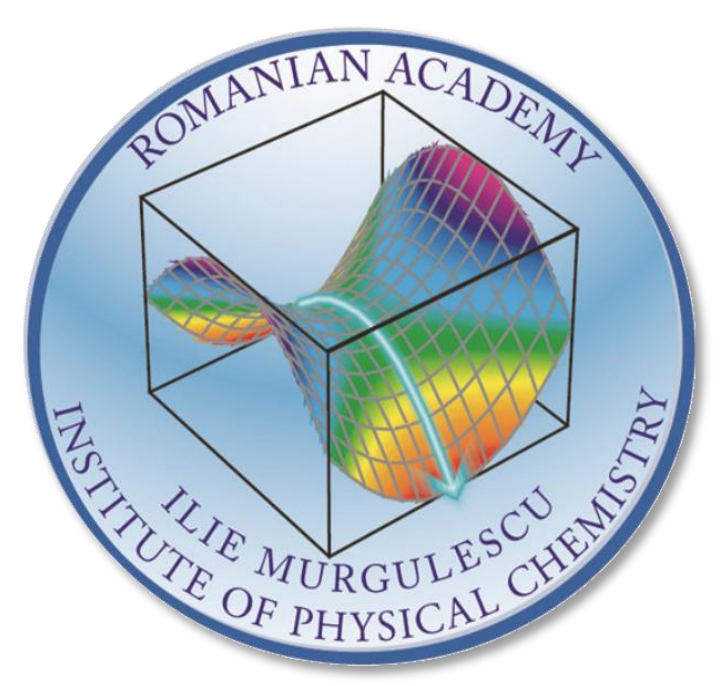

\title{
Interaction studies of some porphyrinic structures with cell membrane using cell suspensions and fluorescent probes
}

\section{Rica Boscencu¹, Georgiana Vasiliu, Gina Manda², Radu Socoteanu³, Dumitru Lupuleasa², Andreea Mihaela Burloiu² Ionela Victoria Neagoe ${ }^{2}$, Laura Olariu ${ }^{4}$ \\ "Carol Davila" University of Medicine and Pharmacy, Faculty of Pharmacy, 6 Traian Vuia St., 020956 Bucharest, Romania 2"Victor Babeş" National Institute of Pathology, 99-101 Splaiul Independentei, 050096 Bucharest, Romania \\ 3"Ilie Murgulescu" Institute of Physical Chemistry, Romanian Academy, 202 Splaiul Independenţei, Bucharest 060021, Romania} 4 "SC. Biotehnos SA", 3-5 Gorunului St., Bucharest, Romania

\section{Abstract}

Porphyrins are tetrapyrrolic structures that have been extensively studied in the recent years, especially for their applicability in the field of theranostic nanomedicine. Selectivity for tumoral tissues manifested by the porphyrins, as well as usage visibile radiations for its activation, implying lower energies compared to those utilised in radiotherapy, are aspects that recommend photodynamic therapy whith porhyrins as efficient alternative treatment to chemotherapy and radiotherapy, with good potential in management of different types of cancer. One of the advantages of simultaneous use of porphyrins in therapeutic and diagnosis purpose, is the fact that, once internalized in the tumor cell, PS becomes an important indicator in monitoring antitumor treatment, the fluorescence signal decreasing with the damage tumour cells [1-4]. The interaction studies with cell membrane can be very useful in highlight of some strategies for designing and optimizing the potential of celular internalization of novel theranostic agents. The aim of the present study was to assess effects exerted in vitro by three unsymmetrical porphyrins (P, Zn(II)P and Cu(II)P), on membrane anisotropy and transmembrane potential of U937

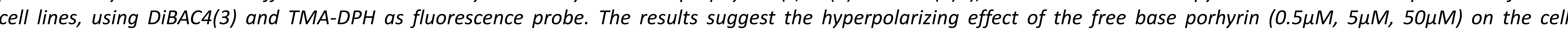
membrane, which recommends its use for therapeutic purposes in low concentrations. The investigated metaloporphyrins have a reduced effect on the membrane potential at $5 \mu \mathrm{M}$ doses, with an increase of $15 \%$ hyperpolarization for $0.5 \mu \mathrm{M}$ and $50 \mu \mathrm{M}$ concentrations, suggesting a dose-dependent mechanism of action.

Keywords: unsymmetrical porphyrins, U937 cells, transmembrane potential, membrane anisotropy

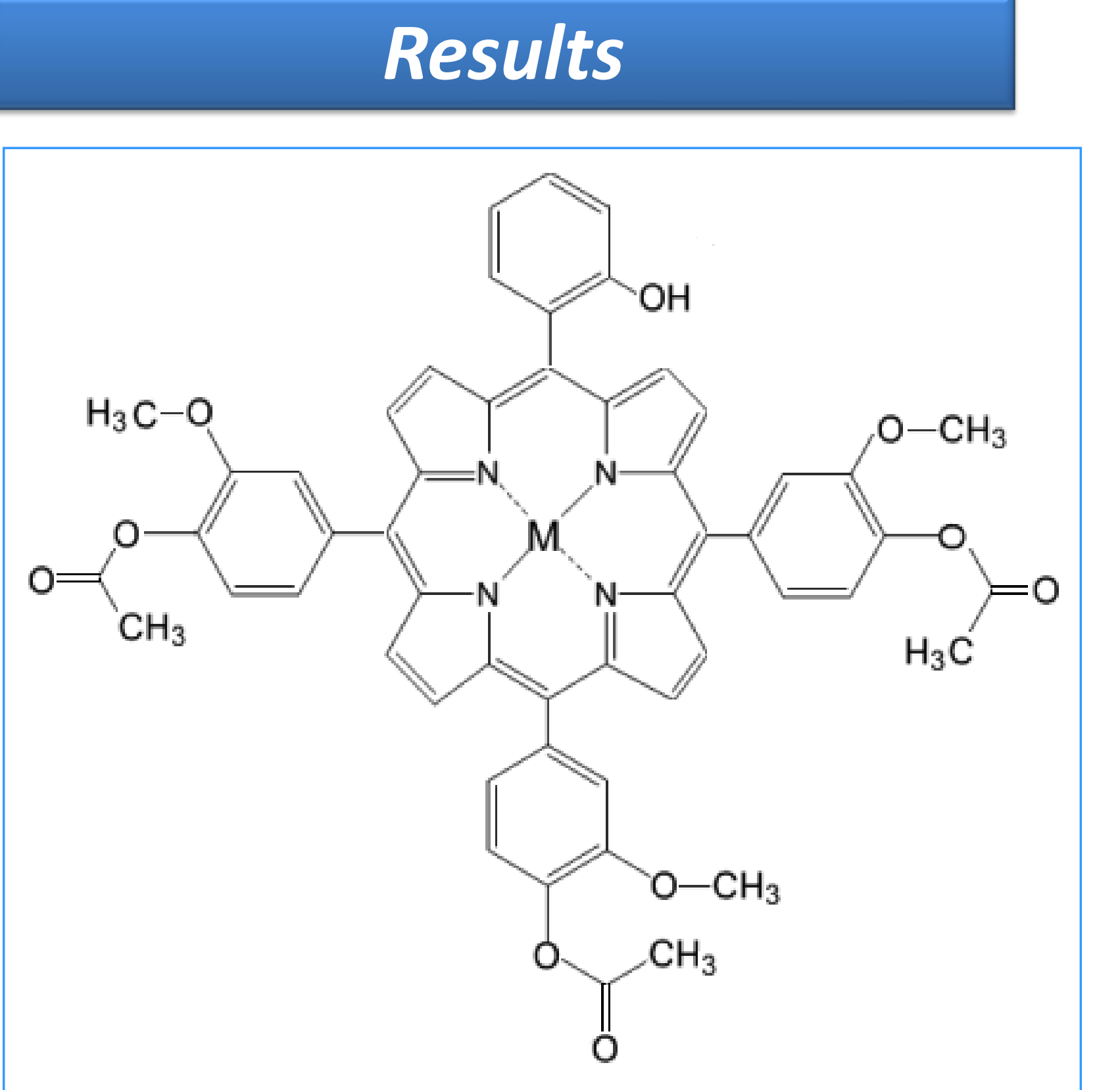

General structures (classic and in silico optimized) of the unsymmetrical porphyrins used in this study $[5,6]$. 5-(2-hydroxyphenyl)-10, 15, 20-tris-(4acetoxy-3-methoxyphenyl)porphyrin, $\mathrm{M}=2 \mathrm{H},($ TMAPOHo), M(II)-5-(2-hydroxyphenyl)-10, 15, 20tris-(4-acetoxy-3methoxyphenyl)porphyrin, $\mathrm{M}=\mathrm{Zn}(\mathrm{II}), \mathrm{Cu}(\mathrm{II})$ (M(II)TMAPOHo)
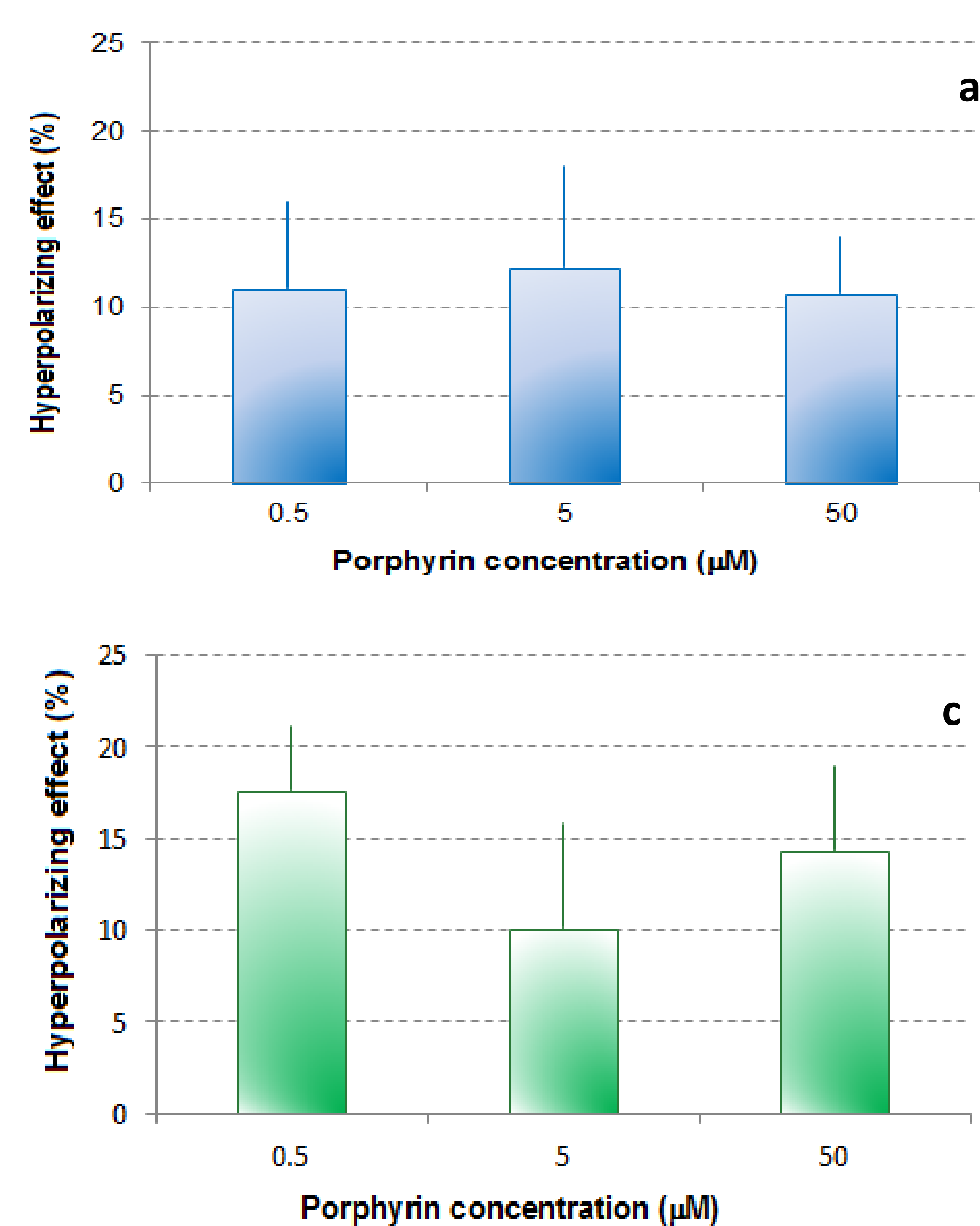

Conclusions
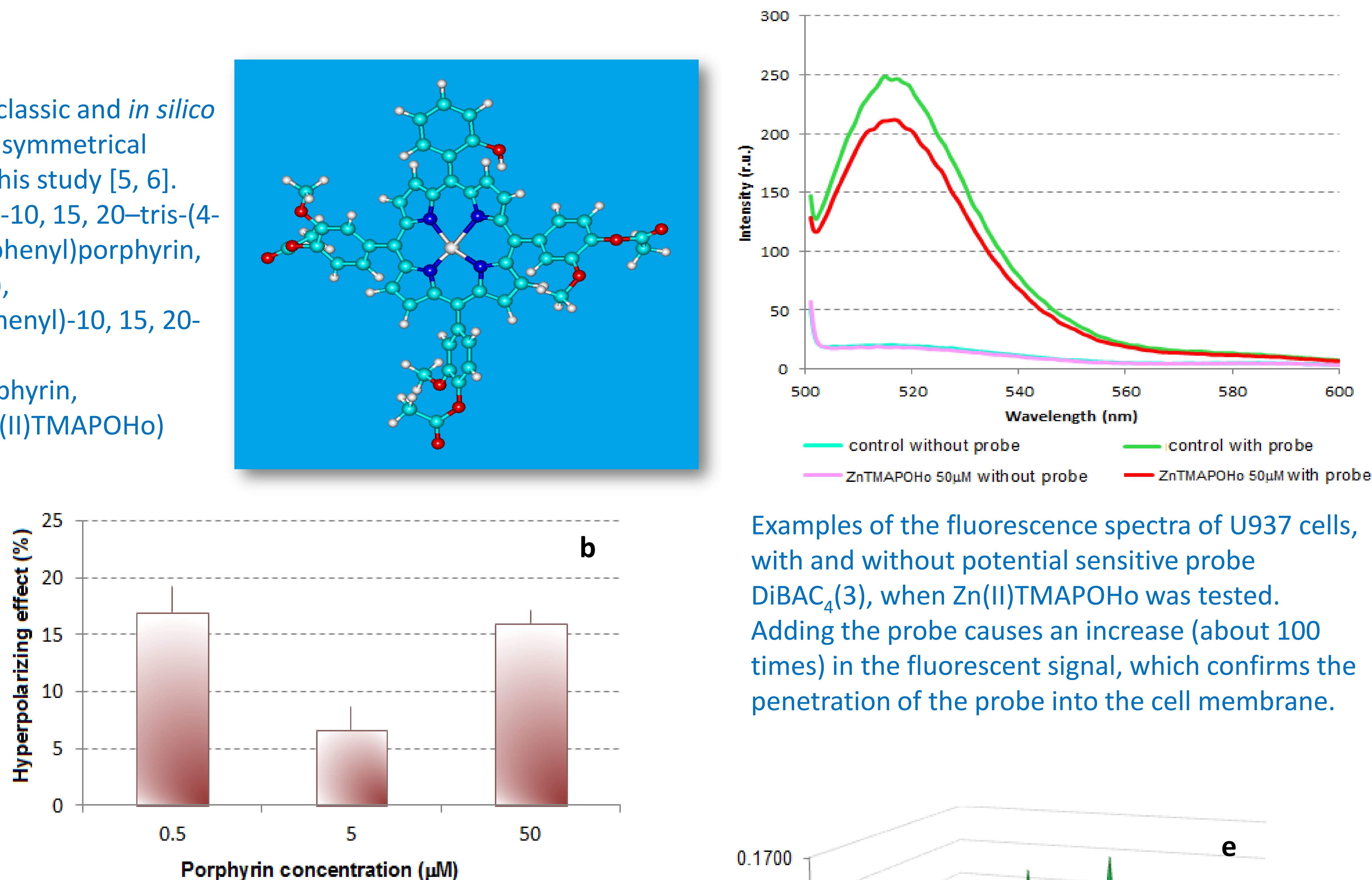

Examples of the fluorescence spectra of U937 cells, with and without potential sensitive probe $\operatorname{DiBAC}_{4}(3)$, when Zn(II)TMAPOHo was tested. Adding the probe causes an increase (about 100 times) in the fluorescent signal, which confirms the penetration of the probe into the cell membrane.

TMAPOHo (a), Zn(II)TMAPOHo (b) and Cu(II)TMAPOHo (c) ffects on the transmembrane potential of U937 cells

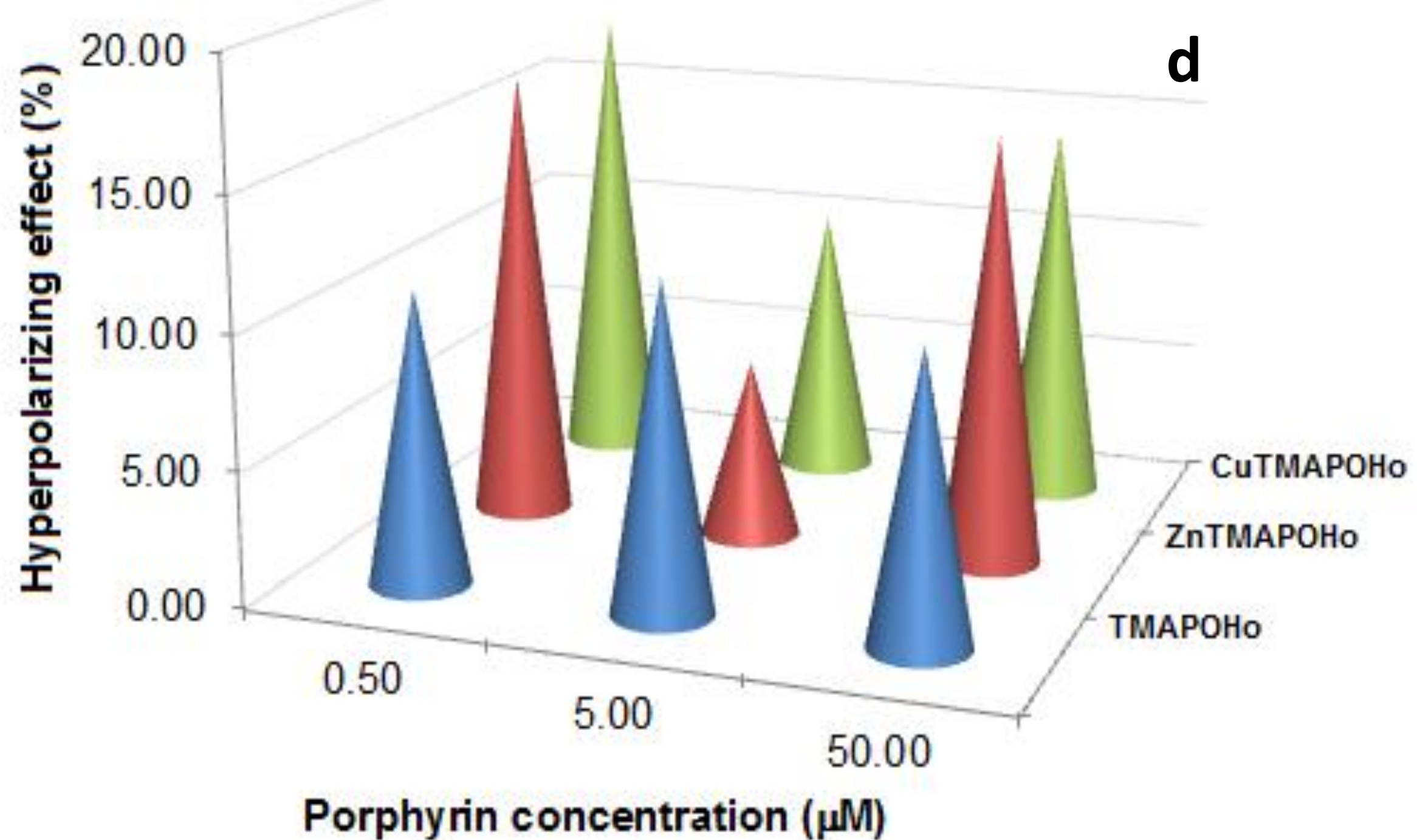

Three unsymmetrical porphyrins are in this study evaluated from the point of view of their effects exerted in vitro on membrane anisotropy and transmembrane potential of U937 cell lines, using DiBAC4(3) and TMA-DPH as fluorescence probes. The obtained results regarding to effect of the tested porphyrins on U937 cell membranes indicates for the free base porphyrin a relatively constant effect, regardless of the dose, while Zn(II) and Cu(II) porphyrins exert a dose-dependent hyperpolarizing effect, suggesting different mechanisms of action. The comparative analysis of the values of the TMA-DPH probe anisotropy coupled to the cell membrane indicates an increased anisotropy in the case of treated cells compared to the control. Considering the fact that the anisotropy is inversely correlated with fluidity, it can be appreciated that membrane of treated cells has becomed more fluid, which means greater freedom of movement of molecules through the phospholipid bilayer. This is a significant result placing the porphyrin in good position for a good active compound in photodynamic further experiments.

\section{References}

1. Sandland J., Malatesti N., Boyle R., Photodiagnosis and Photodynamic Therapy, 2018, 23, 281-294.

2. Zhang J., Jiang C., Longo J. P. F., Azevedo R. B., Zhang H., Muehlmann L. A., Acta Pharmaceutica Sinica B, 2018, 8, 137-146.

3. Sandland J. and Boyle R. W., Bioconjugate Chem., 2019, 30, 975-993.

4. Baskaran R., Lee J., Yang S. G., Biomaterials Research, 2018, 22, 1-8.

5. Boscencu R., Socoteanu R., Vasiliu G., Nacea V., Rev. Chim., 2014, 65, 888-891.

6. Vasiliu G., Boscencu R., Socoteanu R., Nacea V., Rev. Chim., 2014, 65, 998-1001.

Acknowledgments: This study was supported by ERA NET projects: Biomark and Nanother of the Romanian Ministry of Education and Research 